\title{
interview
}

\section{Optical guidance}

\author{
Laser-driven spinning particles can be used to control the direction of nerve fibre growth. Michael Berns \\ from the University of California at Irvine explains this control mechanism and its potential applications.
}

\begin{abstract}
Why is it important to control the direction of nerve fibre growth?

Path-finding in nerve fibres is vital in the development of the nervous system, nerve repair and nerve regeneration. The behaviour of the growth cone at the tip of a growing nerve fibre, an axon, determines the direction of axonal growth and migration. An axon is a highly motile structure that extends filopodia, which sense guidance cues, resulting in a cascade of intracellular signalling that affects the actin and microtubule cytoskeleton and subsequently causes the growth cone to turn towards or away from the guidance cues. Thus, the ability to control the axonal growth direction helps nerve repair and regeneration.
\end{abstract}

\section{What is your work about?}

We have used an optically driven micromotor to control the movement of axons. The optical micromotor uses circularly polarized light, which possesses angular momentum, to trap and spin a birefringent particle, thereby applying a controlled amount of microfluidic shear force to a living cell. In our case, we used retinal ganglion nerve cells from goldfish. We have demonstrated that the growth of a single nerve cell turns in a direction determined by the micromotor spin direction and subsequent microfluidic flow direction. Many previous studies have examined chemotopic/molecular cues that stimulate or repel nerves to grow in one direction or another. However, so far no one has demonstrated that fluidic shear forces can cause a nerve fibre to turn, and that the direction of this turn is determined by the direction of the shear force created by the rotation of the spinning particles. Furthermore, we have discovered that the nerve cell growth cone responds to fluid shear forces of less than a piconewton - around $0.17 \mathrm{pN}$ in our work. Our approach provides a new way to study nerve behaviour and hopefully the internal molecular signalling that occurs once the shear force is applied.

\section{Were there any difficulties?}

There were some technical difficulties. First, the evaluation procedure required uniform particles with the same size and shape,

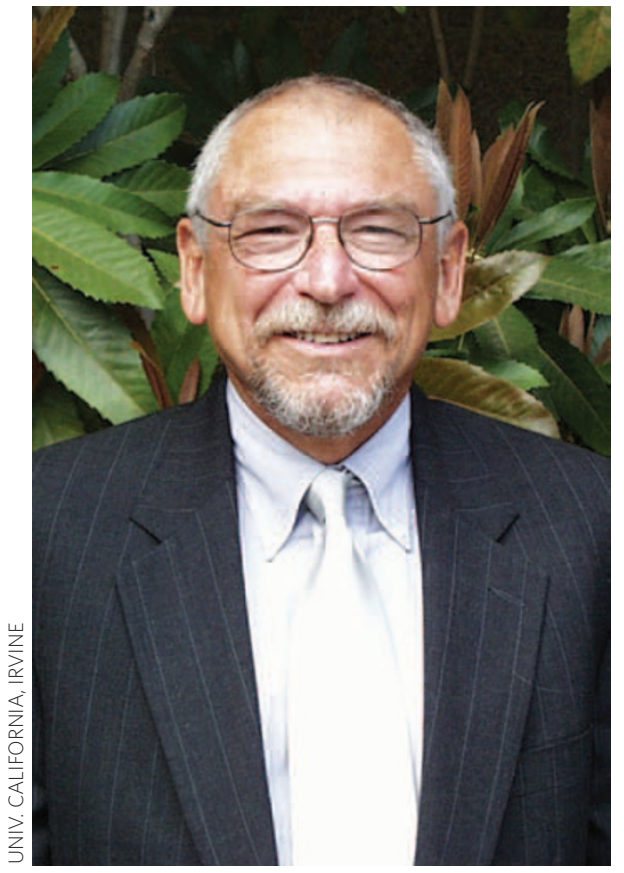

Michael Berns: The success of this multidisciplinary work would have not been possible without the collaboration of biologists, neurobiologists and laser-trapping experts.

and nerve cells of the same age. Second, we didn't know if there was an in vivo corollary to the growth response, or if the cell response was purely in vitro. Third, only $40-50 \%$ of cells responded to the shear force. This may be due to variables such as the age of the nerve culture, variations in the size of the spinning particle, the spinning speed of the particle, and the distance between the particle and the nerve fibre. These variables must be minimized to achieve a high response rate of $70-90 \%$, which will be needed if this technique is to be used for performing meaningful and reliable molecular studies. Although the response rate in our work was not particularly high, we never observed a 'wrong turn' in growth direction with respect to the spinning direction of the birefringent particles - the nerve growth direction either moved in the correct direction or didn't change at all, giving us a statistical significance of less than 0.001 .

\section{What are the potential applications?} Our approach not only offers a new tool for studying axonal growth, but also offers a way to accurately direct axonal growth in vitro, thereby allowing one to connect neurons in specific patterns and build nerve circuits for studying nerve function or for use in bio-electronic devices. Our work also opens up the possibility for developing new ways to repair damaged nerves. Devices containing many microfluidic motors could be transplanted into injured nerves to direct their growth. Although peripheral nerves regenerate easily after minor injuries, they often do not grow back after more serious injuries in which part of the nerve sheath (the large outer wrapping that surrounds the bundles of axons) is missing. The ability to direct nerves back into their sheaths would make successful regeneration much more likely. Our approach also serves as a model system for potential use in other biological systems in which external shear forces play a role in biological functions, such as the cells lining the vascular system, which are very sensitive to the shear forces caused by blood flow, and the extremely sensitive hair cells in the inner ear, which respond to the miniscule forces generated by sound waves.

\section{What are the challenges and future research directions?}

The main challenge is to increase the response percentage. This will boost the reliability of the technique and allow us to look for receptor and signal transduction in the cell membrane, which ultimately causes the cell to turn. We intend to develop a microfluidic chip that allows nerves to be directed down specific channels using microfluidic flows, which would enable repair and regeneration to be studied after selective damage by laser nanoscissors. We also intend to study how nerve growth is affected by particle size and distance from the axon, change the cell model system to one that is more relevant to humans, and embark on molecular studies to improve our understanding of the mechanotransduction signalling.

\section{INTERVIEW BY RACHEL WON}

Michael Berns and co-workers have an Article on controlling the direction of nerve fibre growth on page 62 of this issue. 